pro 100 Patientenjahre, $p=0,14$ ). Außerdem hatten $48 \%$ der Patienten mit endovaskulärer Behandlung, die noch lebten, endograftbezogene Komplikationen und $27 \%$ benötigten Reinterventionen innerhalb der ersten sechs Jahre.

II Kommentar: Die Ergebnisse der bisherigen randomisierten Studien, welche die endovaskuläre mit der offenchirurgischen BAA-Behandlung verglichen, zeigten unisono, dass Erstere die klar weniger invasive, aber auch langfristig weniger erfolgreiche Methode darstellt. Somit könnte man schlussfolgern, dass sich diese Behandlung insbesondere für Patienten mit relevanten Komorbiditäten eignet. Bislang galt je- doch die Meinung, dass BAA-Patienten, die aufgrund ihrer kardiopulmonalen Begleiterkrankungen nicht für einen offenchirurgischen Eingriff geeignet sind, nicht von einer endovaskulären Therapie profitieren und zunächst mit der optimalen Einstellung der Risikofaktoren zu behandeln sind. Dieses Paradigma konnte durch die Langzeitergebnisse der EVAR-2-Studie widerlegt werden. Unabhängig von den relativ hohen Mortalitätsraten dieser Studie konnte gezeigt werden, dass die endovaskuläre Therapie effizient vor BAA-bezogenen Todesfällen schützt.

Außerdem zeigte diese Studie, dass die Möglichkeiten der Sekundärprävention bei diesen Patienten bei Weitem nicht ausge- schöpft wurden:Weniger als die Hälfte der Patienten wurden mit Statinen und Acetylsalicylsäure behandelt.

92\% der befragten Teilnehmer des Charing Cross Meetings gaben an, Patienten, die sich nicht für eine offenchirurgische Sanierung qualifizieren, künftig einer endovaskulären Behandlung zuzuführen.

Dr. med. Nicolas Diehm, Schweizer Herz- und Gefäßzentrum, Inselspital Bern II

II The United Kingdom EVAR trial investigators. Endovascular Repair of Aortic Aneurysm in Patients Physically Ineligible for Open Repair. NEJM 2010 (E-pub ahead of print)

\title{
Blutdruckschwankungen
}

\section{Dagegen helfen am besten Kalziumantagonisten}

\section{Kalziumantagonisten verhindern Schlaganfälle vermutlich deshalb besser als andere Antihypertensiva, weil sie interindividuelle Blutdruck- schwankungen besser abschwächen.}

Die Analyse stützt sich auf randomisierte kontrollierte Interventionsstudien bei Hypertonie. Die Einschlusskriterien erfüllten 398 Studien mit 682 Behandlungsarmen. Ausgewertet wurden die Schwankungen des systolischen Blutdrucks nach einjähriger Behandlung.

Es zeigte sich eine signifikante Heterogenität der Blutdruckvariabilität. Als Ursache hierfür wurde nach der Berücksichtigung verschiedener Einflussfaktoren wie Blutdruckausgangswerte, Alter, Geschlecht, Begleiterkrankungen wie Diabetes und Niereninsuffizienz zu 24,7\% der Typ der Antihypertensiva ausgemacht.

Im Vergleich mit den Gesamtdaten verringerte sich die Blutdruckvariabilität unter Kalziumantagonisten signifikant stärker ( $p<0,0001)$, und unter Betablockern nahm sie signifikant zu ( $p=0,01 ;$ Tab. 1). Auch Diuretika verringerten die Variabilität ( $p=0,006)$, während unter ACE-Hemmern und $\mathrm{AT}_{1}$-Rezeptorantagonisten ungünstige Veränderungen eintraten.

\begin{abstract}
Prinzipiell gleiche Ergebnisse zeigten sich auch bei anderen Analysen, z.B. Antihypertensiva als Erstmedikament, als Kombinationspartner oder in Cross-over-Studien. Die Abnahme der Standardabweichung des systolischen Blutdrucks im Verlauf der Studien geht mit einer Verringerung des Risikos für Schlaganfälle, nicht aber für Herzinfarkte einher.
\end{abstract}

\begin{tabular}{|c|c|}
\hline \multicolumn{2}{|l|}{ Tabelle 1} \\
\hline \multicolumn{2}{|c|}{$\begin{array}{l}\text { Veränderung der Blutdruckvariabilität } \\
\text { unter verschiedenen Antihypertensiva }\end{array}$} \\
\hline $\begin{array}{l}\text { Alle Kalziumantagonisten } \\
\text { Nicht-Dihydropyridin- }\end{array}$ & $-19 \% *$ \\
\hline Kalziumantagonisten & $-19 \%$ \\
\hline Diuretika & $-13 \% *$ \\
\hline ACE-Hemmer & $+8 \%$ \\
\hline $\mathrm{AT}_{1}$-Rezeptorblocker & $+16 \% *$ \\
\hline Betablocker & $+17 \% *$ \\
\hline (* signifikant) & \\
\hline
\end{tabular}

II Kommentar: Die großen Metaanalysen der Hypertonieinterventionsstudien haben für alle Gruppen der Antihypertensiva eindeutig gezeigt, dass das Risiko für Schlaganfälle und Folgen der koronaren Herzkrankheit umso stärker sinkt, je größer der Abfall des systolischen Blutdrucks ist. Im Vergleich der einzelnen Antihypertensiva hat sich als einziger gruppenspezifischer Effekt gezeigt, dass - bei gleicher Blutdrucksenkung - Schlaganfälle durch Kalziumantagonisten besser und durch Betablocker geringer als durch andere Antihypertensiva verhindert werden.

Über die Ursache dieser Unterschiede wurde viel spekuliert. Unbekannte (pleio- trope) Zusatzwirkungen der verschiedenen Substanzen wurden postuliert. Die vorliegende Untersuchung gibt erstmals eine gut begründete, plausible Erklärung für einen substanzspezifischen Effekt der Kalziumantagonisten, der zwar schon bekannt ist, aber bisher keine überzeugende Erklärung fand.

Prof. Dr. med. Heinrich Holzgreve, München

II Webb AJS et al. Effects of antihypertensive-drug class on interindividual variation in blood pressure and risk of stroke: a systematic review and meta-analysis. Lancet 2010;375:906-915 